\title{
Development of Flame Retardant EPDM based Rubber Compounds
}

\author{
Tuğba Akyıldız, Ömer Özdemir, Hümeyra Bozkurt \\ DRC Kauçuk, R\&D Center, \\ Sakarya, Turkey \\ tugba.akyildiz@rubbercompounding.com \\ omer.ozdemir@rubbercompounding.com \\ humeyra@rubbercompounding.com
}

\section{Extended Abstract}

EPDM rubber (Ethylene propylene diene monomer rubber)-based materials can be used up to approximately $150{ }^{\circ} \mathrm{C}$ and have excellent ageing resistance to atmospheric conditions and ozone. These features are advantageous for many applications especially in the automotive industry and hose, electrical cable sheath, gasket and o-ring production.

Due to high filler absorption capacity of EPDM rubber, the cost of materials prepared with EPDM rubber decreases. EPDM rubbers, however, have low fire-safety characteristics and flame resistance of rubber materials EPDM based in many applications need to be improved.

To improve the flame resistance of rubber compounds, halogen, phosphorus containing flame retardants and metal oxide fillers are commonly used by themselves or in combination [1-2]. Because of environmental and health effects of halogen containing chemicals, halogen-free flame retardants are demanded recently [3].

It is well known that by increasing the amount of flame retardant fillers, the flame retardancy improves, but the mechanical properties of the compounds are deteriorated. Any significant decrease in the mechanical properties affect the performance and service life of the rubber material. Therefore, an optimization study is required for both the mechanical properties and flame retardant properties.

In this study, the effects of different flame retardants and metal oxides on the flame resistance property of rubber compounds were investigated. For this purpose, by using two different types of EPDM rubbers, a series rubber compounds containing flame retardant fillers were prepared. The selected flame retarders are antimony trioxide, ammonium polyphosphate (APP) and aluminium hydroxide. For recuperation of negative effects of flame retarders on mechanical properties, some silica and surface treated silica were added to the compounds as reinforcing fillers. Sulfur was used as a vulcanization system. No 8 recipes were studied.

The rubber compounds were prepared with a lab scale internal mixer. Mooney viscosity and rheometer curve of the unvulcanised compounds were measured by using Mooney Viscometer (MV) and Moving Die Rheometer (MDR), respectively. Mechanical testing and hardness, ageing etc. of both unvulcanised compounds and vulcanizates were measured. For mechanical testing the compounds were vulcanized @160 C at a hydraulic press. Then, modulus, tensile strength, elongation at break, hardness and compression set values were measured. The flame resistance of vulcanized compounds is measured with Limiting Oxygen Index (LOI) tester.

To investigate the effects of flame retardants a reference recipe (D1) containing only reinforcing fillers without flame retardants has been created. It has been found that aluminium hydroxide (D2) slightly increased flame resistance. When aluminium hydroxide and antimony trioxide were used together (D3), the flame resistance improved significantly and at the same time mechanical properties also increased with respect to reference recipe. When aluminium hydroxide and APP were used together (D5), a significant increase was also observed, but mechanical properties were worsened. A similar trend was seen also in the case of as in antimony trioxide. The highest flame resistance value was obtained with the combination of aluminium hydroxide, APP and antimony trioxide together (recipe D8). 
In conclusion, flame retardant EPDM-based rubber compounds with different mechanical properties were designed and produced. Thus, EPDM-based compounds demand for various applications in the sector can be met.

\section{References}

[1] I. S. Naumov, S. L. Barbot'ko, A. P. Petrova, and G. V. Malysheva, "The Influence of Flame Retardants on the Properties of Sealing Ethylene-Propylene-Diene Monomer Rubber", Polymer Science, Series D, Vol. 8, No. 2, pp. 110$112,2015$.

[2] Benjamin Zirnstein, Dietmar Schulze, Bernhard Schartel, "Combination of Phosphorous Flame Retardants and Aluminum Trihydrate in Multicomponent EPDM Composites”, Polymer Engineering \& Science, Vol.60, pp 267-280, 2020.

[3] Jong Hwan Do, Do Young Kim and Kwan Ho Seo, "Effect of Eco-friendly Inorganic Flame Retardants on Mechanical and Flame-Retardant Properties of EPDM Compound”, Elastomers and Composites, Vol. 55, No. 1, pp. 40 45, 2020. 